\section{Incidência e fatores de risco para tuberculose em Pelotas, uma cidade do Sul do Brasil}

\author{
Incidence and risk factors for \\ tuberculosis in Pelotas, a city \\ in the South of Brazil
}

Ana M. B. Menezes

Departamento de Clínica Médica Faculdade de Medicina

Universidade Federal de Pelotas (UFPel)

Av. Duque de Caxias, 250 - 96030-002 - Pelotas, RS - Brasil.

\section{Juvenal Dias da Costa}

Departamento de Medicina Social, UFPel.

\section{Helen Gonçalves}

Centro de Pesquisas Epidemiológicas

Departamento de Medicina Social, UFPel.

\section{Saul Morris}

Departamento de Medicina Social, UFPel.

\section{Marcelo Menezes}

\section{Soila Lemos}

Ricardo K.Oliveira

Eduardo Palma

Bolsistas da FAPERGS e CNPq.

\section{Resumo}

Objetivou-se medir a incidência e avaliar fatores de risco para tuberculose, em adultos de Pelotas. Os casos foram detectados na "Secretaria Municipal da Saúde e Bem Estar” (Centro de Saúde), no período de junho de 1994 a junho de 1995. Concomitante, controles populacionais, pareados por sexo e idade, eram sorteados e aplicado o mesmo questionário aos casos e controles. A incidência notificada de tuberculose foi de 72,4/100.000 habitantes. A análise estatística bruta mostrou os seguintes odds ratios: 10,8 (classe social E), 5,4 (renda familiar $\leq 1$ salário mínimo) e 6,6 (analfabetos). O risco em pessoas de cor não branca foi de 4,7; aglomeração e contato com tuberculose apresentaram respectivamente riscos de 3,1 e 5,3. Alcoolismo mostrou um risco de 4,3 e os portadores de doenças associadas à tuberculose um risco de 3,6; as variáveis história de diabete e moradia próxima a pedreiras não se mostraram associadas com tuberculose. Os trabalhadores de pedreiras apresentaram um risco de 4,0. $\mathrm{Na}$ análise por regressão logística condicional, as seguintes variáveis permaneceram, após ajuste para fatores de confusão, significativamente associadas com tuberculose: contato com tuberculose $(\mathrm{OR}=8,2)$, alcoolismo $(\mathrm{OR}=4,0)$, trabalho em pedreira $(\mathrm{OR}=4,7)$ e cor não branca $(\mathrm{OR}=3,1)$. Conclui-se que a incidência de tuberculose em Pelotas é muito elevada e que a maioria dos fatores de risco são passíveis de prevenção ou redução.

Palavras-chave: Tuberculose. Adultos. Incidência. Fatores de risco. 


\section{Abstract}

The aim of this study was to evaluate the incidence and risk factors for tuberculosis, in adults from Pelotas. Cases were recruited in the Sanitary Unit of Tuberculosis (June/1994 to June/1995). At the same time, population controls matched by sex and age were randomly selected, and the same questionnaire was applied for cases and controls. The notified incidence of tuberculosis in Pelotas was $72.4 / 100.000$ inhabitants. Statistical analysis showed the following crude odds ratios: 10.8 (social class E), 5.4 (family income $\leq 1$ minimum salary) and 6.6 (illiterate people). The risk for non-whites was 4.7; crowding and history of contact with tuberculosis presented risks of 3.1 and 5.3, respectively. Alcoholism presented a risk of 4.3 and diseases associated with tuberculosis presented a risk of 3.6; history of diabetes and living near mines did not show an association with tuberculosis. Mine workers had a risk of 4.0. In the multivariate analysis using conditional logistic regression the following risks remained statistically significant after controlling for confounding factors: contact with tuberculosis (8.2), alcoholism (4.0), mine worker (4.7) and non-white (3.1). The incidence of tuberculosis in Pelotas is very high and most risk factors are preventable and can be reduced.

Keywords: Tuberculosis. Adults. Incidence. Risk factors.

\section{Introdução}

Na última década, a tuberculose sofreu um aumento de casos em torno de $20 \%$ em países como os EUA, sendo que em alguns lugares como Nova Iorque, de 1985 a 1992, o aumento foi de $30 \%{ }^{1}$. Em março de 1993, a tuberculose foi declarada pela Organização Mundial da Saúde (OMS) como "emergência mundial de saúde" sendo diversos os fatores atribuídos a este aumento, tais como: surgimento da AIDS, resistência às drogas, piora das condições socioeconômicas e sucateamento dos programas de controle da tuberculose.

A taxa de incidência de tuberculose no Brasil em 1981 foi de 63,4/100.000 habitantes, oscilando de 48,2 a 53,8/100.000 habitantes de 1990 a $1991^{3}$. A análise por regiões fisiográficas mostra importantes diferenças entre os coeficientes de 64,5/ 100.000 habitantes na Região Norte contra 36,2/100.000 habitantes na Região Sul, em 1990. Em 1994, a taxa de incidência de tuberculose de todas as formas, no Rio Grande do Sul (RS), foi de 50,6/100.000 habitantes*.

Pelotas, cidade localizada ao sul do Estado do Rio Grande do Sul, tem apresentado uma das mais altas taxas de incidência de tuberculose em relação a outras cidades do Estado, tendo sido esta em torno de 61,1 e 71,2/100.000 habitantes, nos anos de 1993 e 1994, respectivamente*.

A identificação dos fatores de risco associados à tuberculose é de extrema importância para uma adequada intervenção em termos de saúde pública. O papel dos fatores socioeconômicos na determinação direta da doença ou intermediando outros fatores deve ser identificado. Exposições específicas passíveis de intervenção podem contribuir bastante para o melhor controle da doença. Poucos são os estudos epidemiológicos desenvolvidos no Brasil em que tenham sido investigados tais fatores de risco para tuberculose.

*Informações da Seção de Pneumologia Sanitária, Secretaria da Saúde e Meio Ambiente do Rio Grande do Sul. 


\section{Metodologia}

Realizou-se um estudo de casos e controles para avaliar fatores de risco para tuberculose, em pacientes de 20 a 79 anos, residentes na zona urbana de Pelotas, sendo que a duração do mesmo foi de junho de 1994 a junho de 1995.

Os casos foram identificados como pacientes cujo diagnóstico inicial de tuberculose pulmonar ou extra-pulmonar foi realizado durante o ano em estudo, pela Secretaria Municipal da Saúde e Bem Estar (Centro de Saúde), onde ocorre a centralização do Programa de Controle de Tuberculose, no município de Pelotas. Todos os pacientes com suspeita de tuberculose ou com o diagnóstico já estabelecido são encaminhados ao Centro de Saúde, único local da cidade onde são obtidos os tuberculostáticos. Os exames solicitados para o diagnóstico de tuberculose variaram desde a pesquisa de BK no escarro, reação de Mantoux, radiografia de pulmão até biópsia, quando necessário.

Para cada caso, dois controles pareados por sexo e idade (com amplitude de 5 anos) foram sorteados da população, da seguinte forma: inicialmente, sorteou-se um setor censitário (da zona urbana) dos 250 setores existentes na mesma cidade e, a seguir, um quarteirão com o ponto de início do mesmo; a partir deste ponto, seguindo sempre na mesma direção, todas as casas eram investigadas quanto à presença de um morador que correspondesse ao sexo e à faixa etária do caso. Havendo um morador com estas características, perguntava-se se o mesmo ou alguém na casa era portador de tuberculose, o que seria um critério de exclusão do controle (houve uma exclusão). Para cada novo caso, outros dois controles eram sorteados, seguindo-se a mesma metodologia, iniciando em um novo setor censitário.

O tamanho da amostra alcançado permitiu um poder de $90 \%$, com um intervalo de $95 \%$, para detectar um odds ratio de 3 ; as perdas foram inferiores a $5 \%$ (7 casos e 9 controles).

Um questionário padronizado e pré- codificado foi aplicado aos casos e controles com as seguintes variáveis: sexo, idade, cor (branca ou outra), renda familiar (em salários mínimos), escolaridade (analfabetos, $1^{\circ} \mathrm{e} 2^{\circ}$ graus, nível superior), classe social segundo a classificação proposta pela Associação Brasileira de Institutos de Pesquisa de Mercado (ABIPEME) ${ }^{4}$, aglomeração, trabalho em pedreiras (sim ou não), moradia próxima a pedreiras (sim, para distâncias até $2 \mathrm{~km}$, ou não), história de contato com tuberculose (sim ou não), relato de "história de diabete" (sim ou não) ou de outras doenças (sim ou não) e consumo de álcool.

A classe social segundo a classificação da ABIPEME foi categorizada em quatro níveis: A e B, C, D, E conforme os bens de consumo e escolaridade.

A variável aglomeração foi construída a partir do número de pessoas que dormiam no mesmo quarto: até 2 pessoas, 3 pessoas e 4 pessoas ou mais. "Presença de outras doenças" era uma questão em aberto que, posteriormente, foi dicotomizada: sim (doenças reconhecidamente associadas com tuberculose como AIDS e doenças imunosupressoras) ou não (outras doenças).

O consumo de álcool foi estabelecido através do escore obtido pelo Michigan Alcoholism Screening Test ${ }^{5}$ (MAST) na sua versão simplificada. O questionário para alcoolismo constou de dez perguntas e foi previamente testado em New Orleans e Birmingham em pacientes portadores de tuberculose. Os entrevistadores foram estudantes de medicina.

A análise estatística foi realizada através do Programa SPSS (SPSS Inc. Chicago) ${ }^{6}$ utilizando regressão logística condicional ${ }^{7}$. A análise multivariada foi realizada segundo modelo hierarquizado (Figura). As variáveis socioeconômicas seriam determinantes distais da doença e, portanto, não seriam ajustadas para as demais variáveis. Por outro lado, álcool, história de diabetes, presença de outras doenças, moradia próxima a pedreiras e trabalho em pedreiras seriam determinantes proximais da doença e deveriam ser controladas para as variáveis 
Aglomeração

História de contato com tuberculose

Álcool

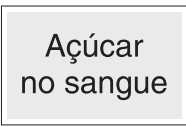

Outras
doenças

\section{Moradia próxima a pedreira}

Trabalho em pedreira

\section{TUBERCULOSE}

Figura - Modelo hierarquizado para tuberculose.

Figure - Tuberculosis hierarchical model.

anteriores do modelo. Aglomeração, por exemplo, poderia estar determinando diretamente a doença ou estar sendo confundida pelas variáveis socioeconômicas.

No modelo hierarquizado, cada bloco de variáveis de um determinado nível é acrescentado na análise e, havendo uma significância estatística $(\leq 0,10)$, estas variáveis permanecem no modelo. Posteriormente, é acrescentado no modelo o bloco de variáveis do nível seguinte.

Um tratamento especial foi dado para a variável cor, com a finalidade de avaliar o componente genético e social da mesma. A análise seguiu dois modelos distintos: em um deles, a variável cor permaneceu no modelo como um determinante distal, no mesmo nível das variáveis socioeconômicas; em outro, cor ingressou no modelo proposto ao nível das variáveis proximais, sendo ajustada para todas as demais variáveis.

\section{Resultados}

Durante o período de junho de 1994 a junho de 1995, foram registrados 192 casos de tuberculose no Centro de Saúde, para todas as idades, na zona urbana de Pelotas. Assim, a incidência notificada de casos novos de tuberculose foi de 72,4/100.000 habitantes. Na faixa etária de 20 a 79 anos, ocorreram 152 casos da doença. Entre estes 152 casos, $72 \%$ foram diagnosticados como portadores de tuberculose pulmonar.

A Tabela 1 descreve as características dos 152 casos e respectivos 295 controles

Tabela 1 - Distribuição de casos e controles em relação à idade e sexo. Pelotas, 1994/95 Table 1 - Distribution of cases and controls according to age and sex. Pelotas, 1994/1995.

\begin{tabular}{lcccc}
\hline Variável & \multicolumn{2}{c}{ Casos } & \multicolumn{2}{c}{ Controles } \\
& $\mathrm{n}$ & $\%$ & $\mathrm{n}$ & $\%$ \\
\hline Idade & & & & \\
20 a 29 anos & 37 & 24,3 & 75 & 25,4 \\
30 a 39 anos & 51 & 33,6 & 93 & 31,5 \\
40 a 49 anos & 33 & 21,7 & 65 & 22,0 \\
50 a 59 anos & 11 & 7,2 & 25 & 8,5 \\
60 a 69 anos & 15 & 9,9 & 27 & 9,2 \\
70 a 79 anos & 5 & 3,3 & 10 & 3,4 \\
Total & 152 & 100,0 & 295 & 100,0 \\
\hline Sexo & & & & \\
Masculino & 105 & 69,1 & 202 & 68,5 \\
Feminino & 47 & 30,9 & 93 & 31,5 \\
Total & 152 & 100,0 & 295 & 100,0 \\
\hline
\end{tabular}


para demonstrar o pareamento por sexo e idade. A maior incidência de casos ocorreu na faixa etária de 20 a 49 anos e no sexo masculino (69\%).

A Tabela 2 mostra a distribuição dos fatores de risco para os casos e controles, os odds ratios brutos com os respectivos intervalos de confiança, além dos testes de significância estatística (para a variável categórica e para tendência linear).

Todas as variáveis socioeconômicas (classe social, renda familiar e escolaridade) estiveram significativa e inversamente associadas com tuberculose ( $\mathrm{p}<0,001)$.

A aglomeração mostrou uma relação direta com tuberculose, na análise bruta. $\mathrm{O}$ risco para tuberculose, em domicílios com quatro ou mais pessoas dormindo na mesma peça, foi cerca de três vezes comparado com domicílios com duas ou menos pessoas. Os indivíduos que relataram contato com pessoas portadoras de tuberculose apresentaram um odds ratio em torno de cinco para doença, quando comparados com os que não tiveram história de contato.

Em relação ao consumo de álcool, verificou-se um odds ratio para tuberculose de 4,31 para as pessoas com alto escore no MAST.

Os indivíduos portadores de doenças reconhecidamente associadas à tuberculose (AIDS e doenças imunossupressoras) apresentaram um risco para tuberculose três vezes maior do que os não portadores destas doenças. História de diabete não mostrou associação significativa com tuberculose.

Também pode ser observado na Tabela 2 que o risco para tuberculose entre os trabalhadores de pedreiras foi quatro vezes maior do que nos demais indivíduos. Não se encontrou significância estatística entre tuberculose e moradia próxima a pedreiras. Quanto à variável cor, o risco para tuberculose em indivíduos de cor não branca comparados com os de cor branca foi de 4,7 .

A Tabela 3 mostra os resultados da análise multivariada por regressão logística realizada segundo modelo hierárquico (Figura). A primeira coluna mostra o odds ratio ajustado para as variáveis socioeconômicas (classe, renda familiar e escolaridade). Ao ser feito este ajuste, observa-se que o odds ratio para aglomeração diminuiu de 3,09 para 1,48, perdendo a significância estatística. Entretanto, as variáveis alcoolismo, doenças associadas, trabalho em pedreira e cor mantiveram a significância estatística, embora com algum decréscimo dos odds ratios. Em relação às variáveis história de diabete e moradia próxima a uma pedreira os resultados permaneceram os mesmos, ou seja, sem associação estatisticamente significativa com tuberculose.

A coluna 2 da Tabela 3 mostra a análise multivariada, conforme o modelo hierarquizado apresentado na Figura. As variáveis socioeconômicas foram mantidas nos modelos 1, 2, 3 e 4 por serem hierarquicamente superiores e estarem altamente associadas com tuberculose. Cor permaneceu nos modelos 1, $2 \mathrm{e} 3$, pois esteve associada significativamente com tuberculose, mesmo após o ajuste para as variáveis socioeconômicas. No modelo 1 , a variável aglomeração foi ajustada para cor e variáveis socioeconômicas e não mostrou associação significativa com tuberculose. No modelo 2, após ajuste para cor e variáveis socioeconômicas, o risco para contato com tuberculose aumentou, o que demonstra que as outras variáveis estavam sendo fatores de confusão negativos. Para a análise do modelo 3, permaneceram no modelo cor, variáveis socioeconômicas e contato com tuberculose, sendo avaliado o efeito das demais. $\mathrm{O}$ risco permaneceu significativo para alcoolismo e trabalho em pedreira.

Como pode ser observado na Tabela 3 , o modelo 4 propõe uma nova posição para a variável cor na determinação da tuberculose. Neste modelo, o efeito desta variável passa a ser avaliado junto com as variáveis proximais, tais como, álcool, história de diabete, outras doenças, moradia próxima a pedreira e trabalho em pedreira, 
Tabela 2 - Distribuição de casos e controles em relação aos fatores de risco. Pelotas, 1994/ 1995.

Table 2 - Distribution of cases and controls according to risk factors. Pelotas, 1994/1995.

\begin{tabular}{|c|c|c|c|c|c|c|c|}
\hline \multirow[t]{2}{*}{ Variável } & \multicolumn{2}{|c|}{ Casos } & \multicolumn{2}{|c|}{ Controles } & \multirow{2}{*}{$\begin{array}{l}\text { Odds } \\
\text { ratio bruto }\end{array}$} & \multirow{2}{*}{$\begin{array}{c}\text { Intervalo de } \\
\text { confiança }\end{array}$} & \multirow[t]{2}{*}{ p-valor } \\
\hline & $\mathrm{n}$ & $\%$ & $\mathrm{n}$ & $\%$ & & & \\
\hline Classe social & & & & & & & $<0,001$ \\
\hline Classe $A+B$ & 9 & 5,9 & 52 & 17,6 & 1,00 & & tendência $<0,001$ \\
\hline Classe C & 16 & 10,5 & 104 & 35,3 & 1,03 & $(0,54-3,57)$ & \\
\hline Classe D & 47 & 30,9 & 91 & 30,8 & 3,82 & $(1,60-9,13)$ & \\
\hline Classe E & 80 & 52,6 & 48 & 16,3 & 10,76 & $(4,42-26,18)$ & \\
\hline Renda familiar & & & & & & & $<0,001$ \\
\hline$>10 \mathrm{SM}$ & 13 & 8,6 & 62 & 21,0 & 1,00 & & tendência $<0,001$ \\
\hline $6,1-10 \mathrm{SM}$ & 12 & 7,9 & 50 & 16,9 & 1,14 & $(0,47-2,77)$ & \\
\hline $3,1-6 \mathrm{SM}$ & 31 & 20,4 & 93 & 31,5 & 1,51 & $(0,71-3,19)$ & \\
\hline $1,1-3 \mathrm{SM}$ & 68 & 44,7 & 70 & 23,7 & 4,12 & $(2,07-8,21)$ & \\
\hline$<=1 \mathrm{SM}$ & 28 & 18,4 & 20 & 6,8 & 5,38 & $(2,33-12,40)$ & \\
\hline Escolaridade & & & & & & & $<0,001$ \\
\hline Nível superior & 6 & 3,9 & 34 & 11,5 & 1,00 & & tendência $<0,001$ \\
\hline $2^{\circ} \mathrm{grau}$ & 16 & 10,5 & 71 & 24,1 & 1,15 & $(0,42-3,20)$ & \\
\hline $1^{\circ} \mathrm{grau}$ & 110 & 72,4 & 172 & 58,3 & 3,37 & $(1,38-8,23)$ & \\
\hline Analfabetos & 20 & 13,2 & 18 & 6,1 & 6,57 & $(2,16-19,97)$ & \\
\hline Aglomeração & & & & & & & 0,005 \\
\hline 2 pessoas & 111 & 73,0 & 246 & 83,4 & 1,00 & & tendência $=0,003$ \\
\hline 3 pessoas & 23 & 15,1 & 35 & 11,9 & 1,56 & $(0,88-2,75)$ & \\
\hline 4 pessoas & 18 & 11,8 & 14 & 4,7 & 3,09 & $(1,41-6,77)$ & \\
\hline \multicolumn{8}{|c|}{ História de contato } \\
\hline com tuberculose & & & & & & & $<0,001$ \\
\hline não & 127 & 83,6 & 285 & 96,6 & 1,00 & & \\
\hline$\underline{\operatorname{sim}}$ & 25 & 16,4 & 10 & 3,4 & 5,31 & $(2,47-11,41)$ & \\
\hline Alcoolismo & & & & & & & $<0,001$ \\
\hline escore baixo & 119 & 78,3 & 277 & 93,9 & 1,00 & & \\
\hline escore alto & 33 & 21,7 & 18 & 6,1 & 4,31 & $(2,25-8,26)$ & \\
\hline Hist.Diabetes & & & & & & & 0,16 \\
\hline Não & 138 & 90,8 & 279 & 94,6 & 1,00 & & \\
\hline$\underline{\operatorname{sim}}$ & 14 & 9,2 & 16 & 5,4 & 1,77 & $(0,79-3,95)$ & \\
\hline Outras doenças & & & & & & & 0,03 \\
\hline Não & 143 & 94,1 & 289 & 98,0 & 1,00 & & \\
\hline sim & 9 & 5,9 & 6 & 2,0 & 3,64 & $(1,10-12,10)$ & \\
\hline \multicolumn{8}{|l|}{ Trabalho } \\
\hline em pedreira & & & & & & & 0,004 \\
\hline Não & 138 & 90,8 & 288 & 97,6 & 1,00 & & \\
\hline$\underline{\operatorname{sim}}$ & 14 & 9,2 & 7 & 2,4 & 4,17 & $(1,59-10,95)$ & \\
\hline \multicolumn{8}{|l|}{ Moradia próxima } \\
\hline a pedreira & & & & & & & 0,18 \\
\hline Não & 138 & 90,8 & 278 & 94,2 & 1,00 & & \\
\hline $\operatorname{sim}$ & 14 & 9,2 & 17 & 5,8 & 1,64 & $(0,80-3,38)$ & \\
\hline Cor & & & & & & & $<0,001$ \\
\hline Branca & 101 & 66,4 & 264 & 89,5 & 1,00 & & \\
\hline não branca & 51 & 33,6 & 31 & 10,5 & 4,74 & $(2,71-8,29)$ & \\
\hline
\end{tabular}


Tabela 3 - Regressão logística para tuberculose conforme modelo hierarquizado (odds ratio e intervalo de confiança de 95\%). Pelotas, 1994/95

Table 3 - Regression logistic for tuberculosis according to a hierarchical model (odds ratio and 95\% confidence interval). Pelotas, 1994/1995.

\section{Variáveis}

Aglomeração

modelo 1

2 pessoas

1,0

1,0

3 pessoas

$1,08(1,04-4,18)$

$1,10(1,29-5,25)$

4 pessoas

$1,48(0,60-3,64)$

$1,22(0,48-3,08)$

$\mathrm{p}$ (categórico)

0,40

0,67

$\mathrm{p}$ (tendência)

0,43

0,65

História de contato

com tuberculose

modelo 2

não 1,0

$1,0 \quad 1,0$

$\operatorname{sim} \quad 8,43(3,29-21,54)$

1,0

p (categórico) $<0,001$

$8,2(3,13-21,32)$

Alcoolismo

escore baixo

1,0

modelo 3

escore alto

$3,63(1,61-8,18)$

1,0

$\mathrm{p}$ (categórico)

0,002

$3,96(1,53-10,27)$

Hist.Diabetes

0,008

não 1,0

modelo 3

sim

1,0

1,0

$\mathrm{p}$ (categórico)

$1,62(0,64-4,09)$

$1,34(0,47-3,79)$

Outras doenças

0,30

0,58

não 1,0

modelo 3

sim

$3,29(0,81-13,40)$

1,0

p (categórico)

0,10

$1,98(0,34-11,53)$

Trabalho em

pedreira

0,45

não
sim

1,0

modelo 3

1,0

p (categórico)

$4,13(1,34-12,71)$

$4,70(1,18-18,76)$

0,01

0,03

Moradia próxima

à pedreira

não

1,0

sim

$1,45(0,60-3,47)$

modelo 3

0,41

1,0

p (categórico)

$1,30(0,45-3,75)$

Cor

branca

1,0

outra

$3,26(1,70-6,26)$

$<0,001$

0,56

modelo 4

1,0

p (categórico)

$<0,001$

$3,11(1,48-6,46)$

0,003

- Modelo hierárquico

Modelo 1 - aglomeração ajustada para cor e variáveis socioeconômicas

Modelo 2 - contato com tuberculose ajustado para cor e variáveis socioeconômicas

Modelo 3 - álcool, diabetes, outras doenças, trabalho em pedreira, moradia perto de pedreira ajustados para cor, variáveis

socioeconômicas e contato com tuberculose

Modelo 4 - cor ajustada para as demais variáveis

- Hierarchical model

Model 1 - Crowding adjusted for colour and socieconomic variables.

Model 2 - History of contact with tuberculosis adjusted for colour and socieconomic variables.

Model 3 - alcohol, diabetes, other diseases, work in mines, living near mines, adjusted for colour, socioeconomic variables and contact with tuberculosis.

Model 4 - colour adjusted for the other variables. 
havendo, portanto, o controle para todas as demais variáveis do modelo. Mesmo após o ajuste, o odds ratio para tuberculose foi cerca de 3 vezes maior para as pessoas de cor não branca.

\section{Discussão}

A incidência notificada de tuberculose é, em Pelotas, de alta magnitude ao ser comparada com outras cidades do RS. Dados oficiais de 1994 apontam a incidência de tuberculose em Pelotas (incluindo zona rural e urbana) como de 71,2/100.000 habitantes, contra 40,4/100.000 em outra cidade do Estado (Sta Rosa)*.

Alguns aspectos metodológicos do presente trabalho merecem ser discutidos. Em relação às perdas do estudo, pode ser dito que as mesmas foram insignificantes. Acredita-se que a totalidade dos casos diagnosticados como tuberculose, na cidade de Pelotas, tenham sido identificados, já que todos os pacientes com este diagnóstico são encaminhados ao Centro de Saúde. Não há outros locais da cidade em que estejam disponíveis os medicamentos tuberculostáticos.

A seleção de controles populacionais parece ter sido apropriada, uma vez que controles de vizinhança levariam a sobreemparelhamento, já que nível socioeconômico é um importante fator de risco. A exclusão de possíveis casos entre os controles sorteados ocorreu sempre que o entrevistado ou alguém naquela residência fosse portador de tuberculose, o que ocorreu uma vez. Em virtude do alto custo que o estudo atingiria, foi inviável submeter os controles a provas laboratoriais que excluíssem tuberculose.

Em relação aos fatores de risco para tuberculose, encontrou-se uma forte associação inversa entre fatores socioeconômicos e tuberculose, como a maioria da literatura aponta ${ }^{8,9}$. Drucker e colaboradores $^{10}$, estudando crianças do Bronx com tuberculose, apontam a pobreza e aglomeração como potentes fatores de risco para tuberculose, mesmo controlando para AIDS. Por outro lado, cabe ressaltar alguns estudos em que tal associação não foi encontrada. Schoeman e colaboradores $^{11}$, na África do Sul, analisaram escolaridade, emprego e condições gerais de habitação, não encontrando associação com tuberculose. Coetzee e colaboradores $^{12}$, também na África do Sul, não encontraram associação entre aglomeração e tuberculose.

A análise multivariada por modelo hierarquizado permitiu avaliar o risco de cada variável controlando para possíveis fatores de confusão. Observa-se facilmente isto ao encontrar-se um risco relativo em torno de três para aglomeração, na análise bruta. Entretanto, observou-se um decréscimo para 1,5 ao controlar para renda familiar, escolaridade e classe social, na análise multivariada. Ficou evidente que o efeito da aglomeração estava sendo confundido pelas variáveis socioeconômicas e que, após o ajuste, tenha desaparecido. O oposto pode ser observado com a variável "história de contato com tuberculose" que, ao ser ajustada para classe social, renda familiar e escolaridade, apresentou um risco relativo maior para doença. Neste caso, os fatores de confusão estavam diminuindo o efeito da associação entre as duas variáveis.

Alcoolismo, assim como tem sido demonstrado na literatura ${ }^{13}$, manteve uma associação significativa com tuberculose que permaneceu mesmo após o controle para as outras variáveis.

Diabetes mellitus tem sido relatado como fator de risco para tuberculose em alguns trabalhos ${ }^{14,15}$, mas são poucos os estudos adequados para que se possa concluir com certeza sobre o risco para tuberculose em diabéticos. AIDS, entretanto, tem sido um dos mais importantes fatores de risco para tuberculose, estudado nos últimos anos ${ }^{16}$. Em países onde o risco de infecção por HIV é alto, como em alguns países da África, o número de casos de tuberculose tem duplicado anualmente ${ }^{17}$. Não se encontrou

* Informações da Seção de Pneumologia Sanitária da Secretaria da Saúde e Meio Ambiente do Rio Grande do Sul. 
associação, no presente estudo, entre relato de "história de diabetes" e tuberculose. Entretanto, a "presença de outras doenças" mostrou associação com tuberculose, talvez pela inclusão de alguns pacientes com AIDS neste grupo. Um estudo de casos e controles, no estado de Washington, foi conduzido para avaliar fatores de risco para tuberculose, comparando as características dos casos com as dos controles obtidos através do censo ${ }^{13}$. $\mathrm{O}$ risco relativo para tuberculose naqueles com infecção por HIV, raça não branca e local de nascimento estrangeiro foi seis vezes maior. Um risco duas vezes maior para tuberculose foi encontrado naqueles com história de doenças associadas, baixo peso para altura, nível socioeconômico inferior, idade superior a 70 anos e alcoolismo.

A associação entre tuberculose e silicose há muito tempo tem sido apontada na literatura. Encontrou-se forte associação entre trabalhadores de pedreiras e tuberculose, mesmo após o ajuste para possíveis fatores de confusão ${ }^{18}$. Em uma coorte de 1153 mineiros, estudada por sete anos, o risco para tuberculose foi de 2,8 (IC $95 \%, 1,9-4,1)^{19}$. Embora este grupo de risco já seja bem reconhecido, pouco se tem feito em relação à quimioprofilaxia do mesmo.

Os resultados da associação entre cor não branca e tuberculose merecem uma maior discussão. A literatura tem demonstrado resultados contraditórios. Stead e colaboradores ${ }^{20}$, por exemplo, encontraram um risco duas vezes maior para tuberculose em adultos da raça negra do que em adultos da raça branca. No entanto, Hoge e colaboradores ${ }^{21}$, estudando crianças escolares, não encontraram risco mais elevado para tuberculose em crianças de raça negra. A variável observada, no presente estudo, foi cor da pele. Tentou-se avaliar uma possível diferença entre as variáveis cor de pele e raça ${ }^{22}$ através de dois modelos de análise em que cor aparece em níveis diferentes de determinação hierárquica. Ao ajustar-se cor para variáveis socioeconômicas, observa-se que o odds ratio, embora com algum decréscimo, mantém a significância estatística. Isto nos leva a pensar que o efeito da cor não branca sobre a tuberculose não se deveu totalmente às condições socioeconômicas adversas. No outro modelo analisado (modelo 4), cor foi ajustada para todas as demais variáveis estudadas, sendo que permaneceu a significância estatística. Parece, portanto, haver um componente genético na cor não branca, além do componente social, que acarreta um maior risco entre essas pessoas para tuberculose. Uma das possíveis explicações para o risco genético da cor é o fato de que, historicamente, essas pessoas entraram em contato com a doença tuberculose posteriormente aos da cor branca e, portanto, não teriam adquirido a mesma resistência ${ }^{23}$.

Deve-se ter em mente que existem algumas limitações para o estudo conclusivo da cor, pois o modelo proposto permite estudar apenas algumas variáveis sociais, quando talvez existam muitas outras.

Concluindo pode-se dizer que a incidência de tuberculose em Pelotas é muito elevada e que os fatores de risco a ela associados são, na sua maioria, passíveis de prevenção ou de redução.

\section{Summary}

A case control study was conducted for identifying risk factors for tuberculosis in patients 20 to 79 years old, living in the urban area of Pelotas, in the south of Brazil, from June 1994 to June 1995. Cases were identified as all new cases diagnosed as pulmonary or extra pulmonary tuberculo- sis by the "Local Tuberculosis Health Service". The notified incidence of tuberculosis was 72.4 new cases per 100.000 inhabitants. The highest frequency was in the 20 to 49 yearold male group (69\%). Conditional logistic regression was used for calculating odds ratios and to adjust each variable to 
the effect of the others. A hierarchical multilevel model was followed. Socioeconomic variables were distal determinants of the disease and therefore were not adjusted for the remainder variables. Crowding was in the second level of the model and history of contact with tuberculosis in the third level. Alcohol, a history of diabetes, living near mines and mine workers were proximal determinants of the disease (fourth level) and were controlled for the variables in the previous level of the model. Each group of variables of a level were entered in the analysis, and variables remained in the model if the statistical significance was $<=0.10$; then, the next block of variables was added to the model. A special treatment was given to the variable colour (white or non-white) in order to evaluate its genetic and social component. The analysis followed two different models: one of them, the variable colour remained in the model as a distal determinant, on the same level as socioeconomic variables; on the other, colour entered the analysis on the level of the proximal variables of the proposed model, being adjusted for all the other variables. Statistical analysis showed the following crude odds ratios: 10.8 (social class E), 5.4 (family income ( 1 minimum salary) and 6.6 (illiterate people). The risk for non-whites was 4.7; crowding and history of contact with tuberculosis showed risks of 3.1 and 5.3, respectively. Alcoholism presented a risk of 4.3 and diseases associated with tuberculosis showed a risk of 3.6; a history of diabetes and living near mines did not show an association with tuberculosis. Mine workers had a risk of 4.0. In the multivariate analysis, using conditional logistic regression, the following risks remained statistically significant after controlling for confounding factors: contact with tuberculosis (8.2), alcoholism (4.0), being a mine worker (4.7) and being non-white (3.1). The incidence of tuberculosis in Pelotas is very high and most of the risk factors are preventable and can be reduced.

\section{Referências}

1. Cantwell MF, Snider DE Jr, Cauthen GM, Onorato IM. Epidemiology of tuberculosis in the United States, 1985 through 1992. JAMA 1994; 272: 535-9.

2. World Health Organization. Tuberculosis: a global emergency. World Health Forum 1993; 14: 438.

3. Hijjar MA. Epidemiologia da tuberculose no Brasil. Inf. Epidemiol SUS 1992 Nov: 53-85.

4. Rutter M. Pesquisa de mercado. São Paulo: Ática; 1988.

5. Bailey WC, Sellers CA, Sutton FD Jr, Sheehy TW, Maetz HM. Tuberculosis and alcoholism. Chest 1978; 73: 183-5.

6. Norussis MJ. SPSS/PC +:Statistical package for social science. [computer program] Chicago:SPSS Inc; 1990.

7. Kirkwood B. Essentials of medical statistics. London: Blackwell Scientific; 1989.

8. Leff A, Lester TW, Addington WW.Tuberculosis: a chemotherapeutic triumph but a persistent socioeconomic problem. Arch Intern Med 1979; 139: 1375-7.
9. Tignor MM. Socioeconomic factors in tuberculosis. N Engl J Med 1981; 304:431.

10. Drucker E, Alcabes P, Bosworth W, Sckell B. Childhood tuberculosis in the Bronx, New York. Lancet 1994; 343:1482-5.

11. Schoeman JH, Westaway MS, Neethling A. The realationship between socioeconomic factors and pulmonary tuberculosis. Int J Epidemiol 1991; 20: 435-40.

12. Coetzee N, Yatch D, Joubert G. Crowding and alcohol abuse as risk factors for tuberculosis in the Mamre population. S Afr Med J 1988; 74: 352-4.

13. Buskin SE, Gale JL, Weiss NS, Nolan CM. Tuberculosis risk factors in adults in King County, Washington, 1988 through 1990. Am J Public Health 1994; 84: 1750-6.

14. Boucot KR, Dillon ES, Cooper DA, et al. Tuberculosis among diabetics. The Philadelphia Survey. Am Rev Tuberc 1952; 65 Suppl:1-50.

15. Opsahl R, Riddervold HO, Aas TW. Pulmonary tuberculosis in mitral stenosis and diabetes mellitus. Acta Tuberc Scand 1961; 40:290-6. 
16. Reichman L. The U-shapped curve of concern [editorial]. Am Rev Respir Dis 1991; 144: 741-2.

17. Ravilglione MC, Snider DE, Kochi A. Global epidemiology of tuberculosis. Morbidity and mortality of a worldwide epidemic. JAMA 1995; 273:220-5.

18. Snider DE Jr. The relationship between tuberculosis and silicosis [editorial].Am Rev Respir Dis 1978; 118: 455-60.

19. Cowie RL. The epidemiology of tuberculosis in gold miners with silicosis. Am J Respir Crit Care Med 1994; 150 (5 part 1): 1460-2.

20. Stead WW, Senner JW, Reddick WT et al. Racial differences in susceptibility to infection by
Mycobacterium tuberculosis. N Engl J Med 1990; 322: $422-7$.

21. Hoge CW, Fisher L, Donnell HD Jr, Dodson DR, Tomlinson GV Jr, Breiman RF, Bloch AB, Good R. Risk factors for transmission of Mycobacterium tuberculosis in a primary school outbreak:lack of racial difference in susceptibility to infection. Am J Epidemiol 1994; 13: 520-30.

22. Cooper R. A note on the biologic concept of race and its application in epidemiologic research. Am Heart J 1984; 108 part 2: 715-23.

23. Cotran RS, Kumar V, Robbins SL. Infectious disease. In: Franz VLT, editors. Robbins pathologic basis of disease. Philadelphia: W.B. Saunders; 1989. p. 307-433. 